\title{
EMBEDDINGS OF FIELDS INTO SIMPLE ALGEBRAS OVER GLOBAL FIELDS*
}

\author{
SHENG-CHI SHIH ${ }^{\dagger}$, TSE-CHUNG YANG ${ }^{\ddagger}$, AND CHIA-FU YU§
}

\begin{abstract}
Let $F$ be a global field, $A$ a central simple algebra over $F$ and $K$ a finite (separable or not) field extension of $F$ with degree $[K: F]$ dividing the degree of $A$ over $F$. An embedding of $K$ into $A$ over $F$ exists implies an embedding exists locally everywhere. In this paper we give detailed discussions about when the converse (i.e. the local-global principle in question) may hold.
\end{abstract}

Key words. Central simple algebras, embeddings, the Hasse principle, Galois cohomology.

AMS subject classifications. 11E72, 17C20.

1. Introduction. The topic on central simple algebras over global fields is a central theme in number theory. One way to extract information of a central simple algebra is to study its maximal subfields. For example, this information would be useful when one attempts to analyze the terms in the geometric side of trace formulas. Indeed, in the geometric side of the trace formula for the multiplicative group of a given central simple algebra, certain major terms (elliptic ones) are described by conjugacy classes of these maximal subfields. This leads to a very important approach, due to Eichler [6], to calculating the class number (of an open compact level subgroup), as the class number can be written as the trace of the characteristic function supported on this level subgroup. In his pioneer work [6] Eichler established results on the trace formula for computing the class numbers and the type numbers of a maximal order or a hereditary order in a given quaternion algebra over any global field. He reduced some of the problem of computing class numbers to computing the so called optimal embeddings among orders in maximal subfields and those in the quaternion algebras, which are of purely local nature. See Eichler [6] and Vignéras [15] for more details.

A useful tool to study maximal subfields is the Hasse principle, which enables us to describe the properties of these subfields from local information. A naive attempt along the same direction is figuring out whether one can describe subfields of a central simple algebra over a global field $F$ from local information. In this paper we study embeddings of a finite field extension of $F$, which does not necessarily have the maximal degree, into a central simple algebra over $F$, and attempted to clarify related problems in this situation. More precisely, consider a finite-dimensional central simple algebra $A$ over a global field $F$ and a finite (separable or not) field extension $K$ of $F$ whose degree divides the degree $\operatorname{deg} A$ of $A$ over $K$, that is, $[K: F] \mid \sqrt{[A: F]}=\operatorname{deg}(A)$. Naturally one considers the following basic questions:

(Q1) What is the necessary and sufficient condition for which the field $K$ can be $F$-linearly embedded into $A$ ?

(Q2) What is the necessary and sufficient condition for which the $F_{v}$-algebra $K_{v}$ can be $F_{v}$-linearly embedded into the $F_{v}$-algebra $A_{v}$ ? Here $v$ is a place of $F$,

\footnotetext{
*Received December 26, 2011; accepted for publication February 22, 2013.

$\dagger$ Institute of Mathematics, Academia Sinica, Astronomy Mathematics Building, No. 1, Roosevelt Rd. Sec. 4, Taipei 10617, Taiwan (r95221018@ntu.edu.tw).

${ }^{\ddagger}$ Department of Mathematics, National Taiwan University, Astronomy Mathematics Building, No. 1, Roosevelt Rd. Sec. 4, Taipei 10617, Taiwan (d97221007@ntu.edu.tw).

§Institute of Mathematics, Academia Sinica and NCTS (Taipei Office), Astronomy Mathematics Building, No. 1, Roosevelt Rd. Sec. 4, Taipei 10617, Taiwan (chiafu@math.sinica.edu.tw).
} 
$F_{v}$ denotes the completion of $F$ at $v, K_{v}:=K \otimes_{F} F_{v}$ and $A_{v}:=A \otimes_{F} F_{v}$.

(Q3) Does the local-global principle for embedding the field $K$ into $A$ as $F$-algebras hold? That is, if $K_{v}$ can be $F_{v}$-linearly embedded in $A_{v}$ for all places $v$ of $F$, then can $K$ be $F$-linearly embedded in $A$ ?

We say that the Hasse principle for a triple $(K, A, F)$ as above holds if the question (Q3) for $K$ and $A$ over a global field $F$ has a positive answer. When the field extension $K$ has the maximal $\operatorname{degree} \operatorname{deg} A$, this result is well known and useful; see Pierce [8, Section 18.4], Prasad-Rapinchuk [9, Proposition A.1] and [10, Proposition 2.7]. However, the case when $K$ does not have the maximal degree is not explored in the literature.

We now outline the contents of this paper. In the first part of this paper we answer the questions (Q1), (Q2) and (Q3). In [17], the third named author of the present paper studies the problem of embeddings of one semi-simple algebra into another one over an arbitrary ground field. In Section 2 we recall some results of embeddings obtained in [17] and provide some proofs of them for the reader's convenience. These give rise to a numerical criterion for the question (Q1) over an arbitrary field; see Lemma 2.4 for the precise statement. In Section 3 we apply results of Section 2 to the case where the base field is either a local field or a global field. This yields a more explicit numerical criterion for (Q1) and (Q2), respectively. Using these criteria, we then show that for any global field $F$, there is a Galois extension $K$ over $F$ of degree 8 and a central simple algebra $A$ over $F$ of degree 24 for which the Hasse principle does not hold; see an example in Section 3.4. Also see Proposition 1.4 below for other cases for which the Hasse principle fails .

We now describe the results in this part. Let $K$ and $A$ be as above. Let $A=$ $\operatorname{Mat}_{n}(\Delta)$, where $\Delta$ is the division part of $A$ and $n:=\mathrm{c}(A)$ is called the capacity of $A$ (see Definition 2.1). Recall the degree $\operatorname{deg}(\Delta)$ of $\Delta$ is $\sqrt{[\Delta: F]}$. For any place $v$ of $F$, let $K_{v}:=K \otimes_{F} F_{v}=\prod_{w \mid v} K_{w}$ be the product of local fields. Let $V^{F}$ and $V^{K}$ denote the sets of places of $F$ and $K$, respectively. Let $d_{v}$ be the order of the class $\left[A_{v}\right]$ in the Brauer group of $F_{v}$, which is also the index of $A_{v}$. By an $F$-embedding of $K$ into $A$ we mean an $F$-linear embedding of $K$ into $A$. The notion of $F_{v}$-embedding of $K_{v}$ into $A_{v}$ is defined similarly.

THEOREM 1.1. Let the notation be as above.

1. The set of $A_{v}^{\times}$-conjugacy classes of $F_{v}$-embeddings of $K_{v}$ into $A_{v}$ is in bijection with the following set

$$
\mathcal{E}_{F_{v}}\left(K_{v}, A_{v}\right):=\left\{\left(x_{w}\right)_{w \mid v} \mid x_{w} \in \mathbb{N}, \sum_{w \mid v} \ell_{w} x_{w}=\mathrm{c}\left(A_{v}\right)\right\},
$$

where

$$
\ell_{w}:=\left[K_{w}: F_{v}\right] / \operatorname{gcd}\left(\left[K_{w}: F_{v}\right], \operatorname{deg}(\Delta)\right) .
$$

In particular, there is an $F_{v}$-embedding of $K_{v}$ into $A_{v}$ if and only if the finite set $\mathcal{E}_{F_{v}}\left(K_{v}, A_{v}\right)$ is non-empty.

2. There is an $F$-embedding of $K$ into $A$ if and only if

$$
[K: F] \mid n \mathrm{c}\left(\Delta \otimes_{F} K\right),
$$

where $\mathrm{c}\left(\Delta \otimes_{F} K\right)$ is the capacity of the central simple algebra $\Delta \otimes_{F} K$ over $K$. 
3. The number $\mathrm{c}\left(\Delta \otimes_{F} K\right)$ can be computed as follows:

(i) The degree $\delta_{0}$ of $\Delta$ is the least common multiple of all $d_{v}^{\prime} s$ for $v \in V^{F}$ (the Hasse-Brauer-Noether theorem).

(ii) For each $w \mid v$, the order $d_{w}^{\prime}$ of the class $\left[A_{v} \otimes_{F_{v}} K_{w}\right]$ in the Brauer group of $K_{w}$ is given by

$$
d_{w}^{\prime}=d_{v} / \operatorname{gcd}\left(\left[K_{w}: F_{v}\right], d_{v}\right) .
$$

(iii) The order $\delta^{\prime}$ of the class $\left[A \otimes_{F} K\right]$ in the Brauer group of $K$ is the least common multiple of $d_{w}^{\prime}$ for all $w \in V^{K}$ (the Hasse-Brauer-Noether theorem).

(iv) The capacity $\mathrm{c}\left(\Delta \otimes_{F} K\right)$ is given by $\mathrm{c}\left(\Delta \otimes_{F} K\right)=\delta_{0} / \delta^{\prime}$.

The proof of Theorem 1.1 is given in Sections 3.1 and 3.2; see especially Propositions $3.2,3.3$ and 3.4.

In the last part of Section 3, we give a necessary and sufficient condition for a pair $(K, A)$ so that the Hasse principle in question holds. Here we fix a global field $F$. We associate to each pair $(K, A)$ an element

$$
\overline{\mathbf{x}}=\left(\overline{\mathbf{x}}_{w}\right)_{w \in V^{K}} \in \bigoplus_{w \in V^{K}} \mathbb{Q} / \mathbb{Z}
$$

as follows. For any $w \in V^{K}$, put

$$
\mathbf{x}_{w}:=\frac{\mathrm{c}\left(A_{v}\right) \cdot \operatorname{gcd}\left(\left[K_{w}: F_{v}\right], d_{v}\right)}{[K: F]} \in \mathbb{Q}_{>0}
$$

and let $\overline{\mathbf{x}}_{w}$ be the class of $\mathbf{x}_{w}$ in $\mathbb{Q} / \mathbb{Z}$, where $v$ is the place of $F$ below $w$.

We show that the element $\overline{\mathbf{x}}$ is an obstruction class to the Hasse principle for $(K, A)$, i.e. if this class does not vanish, then the Hasse principle for $(K, A)$ fails. We also show that the vanishing of $\overline{\mathbf{x}}$ is the only obstruction to the Hasse principle. Namely, we have the following result (Theorem 3.6).

Theorem 1.2. Notations as above. An F-embedding of $K$ into $A$ exists if and only if an $F_{v}$-embedding of $K_{v}$ into $A_{v}$ exists for all $v \in V^{F}$ and that the element $\overline{\mathbf{x}}$ vanishes.

The second part of this paper deals with the Hasse principle for a family of pairs $(K, A)$ over a fixed global field $F$ with both degrees $[K: F]$ and $\operatorname{deg}(A)$ constant. Let $(k, \delta)$ be a pair of positive integers with $k \mid \delta$. We say that the Hasse principle for the pair $(k, \delta)$ holds if for any finite field extension $K$ over $F$ of degree $k$ and any central simple algebra $A$ over $F$ of degree $\delta$, the Hasse principle for the pair $(K, A)$ holds.

We describe the results in this part. Let $(k, \delta)$ be a pair of positive integers as above. For each partition $\lambda$ of $k$

$$
\lambda=\left(k_{1}, \ldots, k_{t}\right), \quad k_{1} \leq \cdots \leq k_{t}, \quad \sum_{i=1}^{t} k_{i}=k
$$

and a positive divisor $s \mid \delta$, we define a finite set

$$
\mathcal{E}(\lambda, s):=\left\{\left(x_{i}\right) \in \mathbb{N}^{t} \mid \sum_{i=1}^{t} \ell_{i} x_{i}=s\right\},
$$


where $\ell_{i}:=k_{i} / \operatorname{gcd}\left(k_{i}, d\right)$, and a vector $\mathbf{x}(\lambda, s)$ in $\mathbb{Q}^{t}$, where

$$
\mathbf{x}(\lambda, s):=\left(\mathbf{x}_{i}\right), \quad \mathbf{x}_{i}=\mathbf{x}_{i}(\lambda, s):=s \operatorname{gcd}\left(k_{i}, d\right) / k, \quad \forall i=1, \ldots, t .
$$

Let $\operatorname{LD}(k, \delta)$ be the set consisting of all pairs $(\lambda, s)$, where

- $\lambda$ is a partition of $k$, and

- $s$ is a positive integer dividing $\delta$,

such that the set $\mathcal{E}(\lambda, s)$ is non-empty. ("LD" stands for all possible local decompositions). The following result gives a simple way to check the Hasse principle for a pair $(k, \delta)$.

THEOREM 1.3. Let $(k, \delta)$ be a pair of positive integers with $k \mid \delta$.

(1) Suppose for all elements $(\lambda, s) \in \operatorname{LD}(k, \delta)$, the vector $\mathbf{x}(\lambda, s)$ has integral components. Then the Hasse principle for $(k, \delta)$ holds.

(2) Suppose there is an element $(\lambda, s) \in \operatorname{LD}(k, \delta)$ such that $\mathbf{x}_{i}(\lambda, s) \notin \mathbb{N}$ for some $i$. Then there is a finite separable field extension $K$ of degree $k$ over $F$ and $a$ central division algebra $A$ of degree $\delta$ over $F$ so that the Hasse principle for $(K, A)$ fails. In particular, the Hasse principle for the pair $(k, \delta)$ fails.

The proof of Theorem 1.3 uses Theorem 1.2, the Hilbert irreducibility theorem and the global class field theory. Based on Theorem 1.3, we prove the following result (Proposition 4.6 and Corollary 4.7), which also shows that (Q3) has a negative answer in general.

\section{Proposition 1.4.}

1. Let $\delta=p_{1}^{n_{1}} \cdots p_{r}^{n_{r}}$, and let $k$ be a positive integer with $k>1$ and $k \mid \delta$, where $p_{i}^{\prime}$ s are distinct prime divisors of $\delta$. Assume that $k \leq \delta / p_{i}^{n_{i}}$ and $p_{i} \mid k$ for some $1 \leq i \leq r$ (so $r \geq 2$ ). Then the Hasse principle for the pair $(k, \delta)$ does not hold.

2. Let $\delta$ be a positive integer divisible by at least two primes. Then there is a positive integer $k$ with $k \mid \delta$ such that the Hasse principle for the pair $(k, \delta)$ does not hold.

Clearly Proposition 1.4 (2) follows from Proposition 1.4 (1). Proposition 1.4 tells us that when $\delta$ is divisible by two primes and $k$ is "small" comparable to $\delta$, the Hasse principle for $(\delta, k)$ fails.

In the last section we discuss the Hasse principle for a geometric orbit of the variety of embeddings of $K$ into $A$ that is defined over $F$, where $K$ is a finite etale commutative $F$-algebra. Let $X$ be the $F$-scheme of embeddings of $K$ into $A$ (see Section 5). The group $H=\mathrm{GL}_{1}(A)$ acts on $X$ by conjugation. We have the following result.

TheOREM 1.5 (The Hasse principle). Let $\bar{X}_{0}$ be a geometric orbit of $H \otimes_{F} F^{\text {sep }}$ that is defined over $F$ and let $X_{0}$ be the $F$-subscheme of $X$ whose base extension to $F^{\text {sep }}$ is $\bar{X}_{0}$. If $X_{0}\left(F_{v}\right) \neq \emptyset$ for all $v \in V^{F}$, then $X_{0}(F) \neq \emptyset$.

The proof relies on results of Springer [14], Douai and Borovoi [1] about the second non-abelian Galois cohomology and neutral classes. Among early results in this area one can mention the validity of the Hasse principle for projective homogeneous spaces due to Harder [7] and for symmetric homogeneous spaces of absolutely simple simply-connected groups due to Rapinchuk [11]. Later, Borovoi in a series of papers developed cohomological methods for analyzing the Hasse principle for homogeneous spaces with connected stabilizers, of an arbitrary connected group 
whose maximal semi-simple subgroup is simply-connected; see Borovoi $[2,3]$. In [10, Appendix A] Prasad and Rapinchuk used the cohomological method to study the local-global principle for embeddings of maximal subfields into central simple algebras over global fields with involutions.

This paper is organized as follows. In Section 2 we collect and show some general embedding results over any field based on [17]. In Section 3, we give a more detailed study about embeddings of a field extension $K$ into a central simple algebra $A$ over a global field. We determine for which pair $(K, A)$ the Hasse principle holds. In particular, we answer the questions (Q1), (Q2) and (Q3). In Section 4, we work on the Hasse principle for a given pair $(k, \delta)$ of positive integers with $k$ dividing $\delta$. In Section 5 we give the proof of Theorem 1.5 following a referee's suggestion.

\section{General embedding results.}

2.1. Setting. Let $F$ denote the ground field, which is arbitrary in this section. All $F$-algebras considered in this paper are assumed to be finite-dimensional as $F$-vector spaces and have the identity. As the standard convention, an $F$-algebra homomorphism is a ring homomorphism over $F$; in particular, it sends the identity of the source to the identity of the target.

We recall the following definition for central simple algebras; see [12].

Definition 2.1. The degree, capacity, and index of a central simple algebra $A$ over $F$ are defined as

$$
\operatorname{deg}(A):=\sqrt{[A: F]}, \quad \mathrm{c}(A):=n, \quad \mathrm{i}(A):=\sqrt{[\Delta: F]},
$$

if $A \cong \operatorname{Mat}_{n}(\Delta)$, where $\Delta$ is a division algebra over $F$, which is uniquely determined by $A$ up to isomorphism. The algebra $\Delta$ is also called the division part of $A$.

For the convenience of discussion, we introduce the following definition and notations.

DEFINITION 2.2.

1. Let $V$ be a finite-dimensional vector space over $F$, and $A$ a finite-dimensional arbitrary $F$-algebra. We say that $V$ admits an $A$-module structure if there is a right (or left) $A$-module structure on $V$. If $B$ is any $F$-subalgebra of $A$ and $V$ is already a right (resp. left) $B$-module, then by saying $V$ admits an $A$-module structure we mean that the right (resp. left) $A$-module structure on $V$ is required to be compatible with the underlying $B$-module structure on $V$.

2. For any two $F$-algebras $A_{1}, A_{2}$, let $\operatorname{Hom}_{F}\left(A_{1}, A_{2}\right)$ denote the set of $F$-algebra homomorphisms from $A_{1}$ to $A_{2}$, and let

$$
\operatorname{Hom}_{F}^{*}\left(A_{1}, A_{2}\right) \subset \operatorname{Hom}_{F}\left(A_{1}, A_{2}\right)
$$

be the subset consisting of embeddings of $A_{1}$ into $A_{2}$. For two maps $\varphi_{1}, \varphi_{2} \in$ $\operatorname{Hom}_{F}\left(A_{1}, A_{2}\right)$, we say $\varphi_{1}$ and $\varphi_{2}$ are equivalent if there is an element $b \in$ $A_{2}^{\times}$such that $\varphi_{2}=\operatorname{Int}(b) \circ \varphi_{1}$. That is, $\varphi_{2}(a)=b \varphi_{1}(a) b^{-1}$ for all $a \in$ $A_{1}$. Then $A_{2}^{\times} \backslash \operatorname{Hom}_{F}\left(A_{1}, A_{2}\right)$ is the set of equivalence classes of $F$-algebra homomorphisms from $A_{1}$ to $A_{2}$. Write

$$
\mathcal{O}_{A_{1}, A_{2}}:=A_{2}^{\times} \backslash \operatorname{Hom}_{F}\left(A_{1}, A_{2}\right) \quad \text { and } \quad \mathcal{O}_{A_{1}, A_{2}}^{*}:=A_{2}^{\times} \backslash \operatorname{Hom}_{F}^{*}\left(A_{1}, A_{2}\right)
$$


for the orbits spaces.

We often simply write $A_{1} \otimes A_{2}$ for $A_{1} \otimes_{F} A_{2}$ if the ground field $F$ is understood.

2.2. General embedding lemmas. Let $A$ be a central simple algebra over $F$. We realize $A$ as $\operatorname{End}_{\Delta}(V)$, the endomorphism algebra of $V$, where $\Delta$ is the division part of $A$ and $V$ is a right $\Delta$-module of finite rank. Let $A^{\prime}$ be another simple $F$-algebra with center $K$. Then there is an $F$-algebra homomorphism $\varphi: A^{\prime} \rightarrow A$ if and only if there is an $\left(A^{\prime}, \Delta\right)$-bimodule structure on $V$, or $V$ admits a right $\Delta \otimes_{F} A^{\prime o}$-module structure. Here $A^{\prime o}$ denotes the opposite algebra of $A^{\prime}$. Suppose $n:=\operatorname{dim}_{\Delta} V$ and

$$
\Delta \otimes_{F} A^{\prime o} \simeq\left(\Delta \otimes_{F} K\right) \otimes_{K} A^{\prime o} \simeq \operatorname{Mat}_{c}\left(\Delta^{\prime}\right),
$$

where $\Delta^{\prime}$ is the division part of the central simple algebra $\Delta \otimes_{F} A^{\prime o}$ over $K$. We have

$$
[\Delta: F]\left[A^{\prime}: F\right]=c^{2}\left[\Delta^{\prime}: F\right] \quad \text { and } \quad \operatorname{dim}_{F} V=n[\Delta: F] .
$$

Then $V$ admits a right $\Delta \otimes_{F}{A^{\prime}}^{\circ}$-module structure if and only if

$$
c\left[\Delta^{\prime}: F\right] \mid \operatorname{dim}_{F} V .
$$

This is equivalent to $[\Delta: F]\left[A^{\prime}: F\right] \mid c n[\Delta: F]$, or equivalently

$$
\left[A^{\prime}: F\right] \mid n c \text {. }
$$

We have proved the following result.

Lemma 2.3. Let $A$ be a central simple $F$-algebra and $A^{\prime}$ a simple $F$-algebra. Suppose $A \simeq \operatorname{Mat}_{n}(\Delta)$. Then there exists an $F$-embedding of $A^{\prime}$ into $A$ if and only if

$$
\left[A^{\prime}: F\right] \mid n \cdot \mathrm{c}\left(\Delta \otimes_{F} A^{\prime o}\right) .
$$

Next we consider the case where $A^{\prime}$ is a semi-simple $F$-algebra instead. Write $A^{\prime}=\prod_{i=1}^{s} A_{i}^{\prime}$ into simple factors and let $K_{i}$ be the center of $A_{i}^{\prime}$. The existence of an $F$-embedding of $A^{\prime}$ into $A$ is equivalent to that there is an $\left(A^{\prime}, \Delta\right)$-bimodule structure on $V$. This means that there is a decomposition of $V$ into $\Delta$-submodules

$$
V=V_{1} \oplus \cdots \oplus V_{s}
$$

so that each $V_{i}$ is a non-zero $\left(A_{i}^{\prime}, \Delta\right)$-bimodule. Put $n_{i}:=\operatorname{dim}_{\Delta} V_{i}$ and let $c_{i}$ be the capacity of the central simple algebra

$$
\Delta \otimes_{F} A_{i}^{\prime o}=\left(\Delta \otimes_{F} K_{i}\right) \otimes_{K_{i}} A_{i}^{\prime o}
$$

over $K_{i}$. Then we get the conditions

$$
n=\sum_{i=1}^{s} n_{i} \quad \text { and } \quad\left[A_{i}^{\prime}: F\right] \mid n_{i} c_{i}, \quad \forall i=1, \ldots, s .
$$

This yields the following criterion for embeddings.

Lemma 2.4. Let $A$ and $A^{\prime}=\prod_{i=1}^{s} A_{i}^{\prime}$ be as above. Then there is an embedding of the $F$-algebra $A^{\prime}$ into $A$ if and only if there are positive integers $n_{i}$ for $i=1, \ldots, s$ such that

$$
n=\sum_{i=1}^{s} n_{i} \quad \text { and } \quad\left[A_{i}^{\prime}: F\right] \mid n_{i} c_{i}, \quad \forall i=1, \ldots, s,
$$


where $c_{i}$ the capacity of the central simple algebra $\Delta \otimes_{F} A_{i}^{\prime o}$ over $K_{i}$.

Lemma 2.5. Let $A$ and $A^{\prime}=\prod_{i=1}^{s} A_{i}^{\prime}$ be as above. Let $\varphi, \varphi^{\prime}$ be two maps in $\operatorname{Hom}_{F}\left(A^{\prime}, A\right)$, and let $V_{\varphi}$ and $V_{\varphi^{\prime}}$ be the associated $\left(A^{\prime}, \Delta\right)$-bimodules underlying the space $V$. Then $\varphi$ and $\varphi^{\prime}$ are equivalent if and only if the $\left(A^{\prime}, \Delta\right)$-bimodules $V_{\varphi}$ and $V_{\varphi^{\prime}}$ are isomorphic.

Proof. See [17, Lemma 3.2] प

2.3. Maximal degree field extension case. We apply Lemmas 2.3 and 2.4 to the case where the semi-simple algebra $A^{\prime}$ is commutative and obtain the following well-known result. This is also a consequence of a result of Chuard-Koulmann and Morales [5, Proposition 4.3].

\section{LEMMa 2.6 .}

1. Let $A$ be a central simple algebra over $F$ and $K$ is a field extension of $F$ with $[K: F]=\operatorname{deg}(A)$. Then there exists an $F$-embedding of $K$ into $A$ if and only if $K$ splits $A$.

2. Let $A$ be a central simple algebra over $F$ and $K=\prod_{i=1}^{s} K_{i}$ is commutative semi-simple $F$-algebra with $[K: F]=\operatorname{deg}(A)$. Then there exists an $F$ embedding of $K$ into $A$ if and only if each $K_{i}$ splits $A$.

Proof. (1) By Lemma 2.3, the set $\operatorname{Hom}_{F}(K, A)$ is non-empty if and only if [ $K$ : $F] \mid n c$, where $c:=c(\Delta \otimes K)$. If $K$ splits $\Delta$, then $c=\operatorname{deg}(\Delta)$ and hence $[K: F]=$ $n \operatorname{deg}(\Delta)=n c$. Therefore, $\operatorname{Hom}_{F}(K, A)$ is non-empty. Suppose $[K: F] \mid n c$. Then $\operatorname{deg}(\Delta) \mid c$ and $\operatorname{deg}(\Delta)=c$. This shows that $K$ splits $\Delta$.

(2) Suppose there is an $F$-embedding of $K$ into $A$. Then there are positive integers $n_{i}$ with $n=\sum n_{i}$ and there is an embedding of $K_{i}$ into $\operatorname{Mat}_{n_{i}}(\Delta)$. Since $\left[K_{i}: F\right] \mid n_{i} \operatorname{deg}(\Delta)$, it follows from

$$
[K: F]=\operatorname{deg}(A)=\sum_{i} n_{i} \operatorname{deg}(\Delta) \geq \sum_{i}\left[K_{i}: F\right]=[K: F]
$$

that $\left[K_{i}: F\right]=n_{i} \operatorname{deg}(\Delta)$ for each $i=1, \ldots, s$. Therefore, $K_{i}$ splits $\Delta$. Conversely, if $K_{i}$ splits $\Delta$ for each $i$, then $\left[K_{i}: F\right]=m_{i} \operatorname{deg}(\Delta)$ for a positive integer $m_{i}$ and $c_{i}:=\mathrm{c}\left(\Delta \otimes_{F} K_{i}\right)=\operatorname{deg}(\Delta)$. Then we have

$$
n=\sum_{i} m_{i}, \quad \text { and } \quad\left[K_{i}: F\right] \mid m_{i} c_{i}, \quad \forall i=1, \ldots, s .
$$

It follows from Lemma 2.4 that there is an embedding of $K$ into $A$.

3. Answers to (Q1) and (Q2) by numerical invariants. In this section we study $F$-embeddings of $K$ into $A$ over $F$, where $F$ is either a local or global field, $K$ is a commutative semi-simple algebra over $F$ and $A$ is a central simple algebra.

3.1. Local results. Let $F$ denote a local field.

Lemma 3.1. Let $A=\operatorname{End}_{\Delta}(V)=\operatorname{Mat}_{n}(\Delta)$ be a central simple algebra over $F$.

1. Let $K$ be a finite field extension of $F$. The following statement are equivalent:

(a) There exists an embedding of $K$ into $A$ over $F$.

(b) $[K: F] \mid n \cdot \mathrm{c}\left(\Delta \otimes_{F} K\right)$.

(c) $[K: F] \mid n \operatorname{deg}(\Delta)$. 
2. Let $K=\prod_{i=1}^{s} K_{i}$ be a commutative semi-simple algebra over $F$. Then there exists an embedding of $K$ into $A$ over $F$ if and only if there are positive integers $n_{i}$ for $i=1, \ldots, s$ such that

$$
n=\sum_{i=1}^{s} n_{i} \quad \text { and } \quad\left[K_{i}: F\right] \mid n_{i} \operatorname{deg}(\Delta), \quad \forall i=1, \ldots, s .
$$

It follows from Lemma 2.4 that the statements (a) and (b) are equivalent. The implication $(b) \Longrightarrow(c)$ is trivial. Put $\delta:=\operatorname{deg}(\Delta)$ and $k:=[K: F]$. If $\operatorname{inv}(\Delta)=a / \delta$ with $\operatorname{gcd}(a, \delta)=1$, then (see [13])

$$
\operatorname{inv}\left(\Delta \otimes_{F} K\right)=[K: F] \operatorname{inv}(\Delta)=\frac{a k}{\delta}=\frac{a^{\prime}}{\delta^{\prime}}, \quad \text { with } \operatorname{gcd}\left(a^{\prime}, \delta^{\prime}\right)=1,
$$

where $\delta=\delta^{\prime} c, a k=a^{\prime} c$, and $c:=\operatorname{gcd}(k, \delta)$. It follows that

$$
\mathrm{c}\left(\Delta \otimes_{F} K\right)=\operatorname{gcd}([K: F], \operatorname{deg}(\Delta)) .
$$

Note that $\operatorname{gcd}\left(\delta^{\prime}, k\right)=1$, so we have

$$
k|n \delta \Longleftrightarrow k| n c \delta^{\prime} \Longleftrightarrow k \mid n c .
$$

The statement Lemma 3.1 (2) follows from Lemma 2.4 and Lemma 3.1 (1).

Now consider the case where $K=\prod_{i=1}^{s} K_{i}$ is a commutative semi-simple $F$ algebra. Put

$$
c_{i}:=\operatorname{gcd}\left(\left[K_{i}: F\right], \operatorname{deg}(\Delta)\right) \quad \text { and } \quad \ell_{i}:=\left[K_{i}: F\right] / c_{i} .
$$

For any positive integer $n_{i}$, we have

$$
\left[K_{i}: F\right]\left|n_{i} \operatorname{deg}(\Delta) \Longleftrightarrow \ell_{i}\right| n_{i} .
$$

Put

$$
\mathcal{E}_{F}(K, A):=\left\{x=\left(x_{1}, \ldots, x_{s}\right) \in \mathbb{N}^{s} \mid \sum_{i=1}^{s} \ell_{i} x_{i}=\operatorname{dim}_{\Delta} V\right\} .
$$

If a tuple $\mathbf{n}=\left(n_{1}, \ldots, n_{s}\right)$ is a solution to (3.1), then the tuple $x=\left(x_{1}, \ldots, x_{s}\right)$, where $x_{i}:=n_{i} / \ell_{i}$, is an element in $\mathcal{E}_{F}(K, A)$. Conversely, any element $x$ in $\mathcal{E}_{F}(K, A)$ gives a solution $\mathbf{n}$ to (3.1) by setting $n_{i}=\ell_{i} x_{i}$. Recall that $A^{\times} \backslash \operatorname{Hom}_{F}^{*}(K, A)$ is the set of equivalence classes of embeddings of $F$-algebras from $K$ into $A$.

Proposition 3.2. There is a natural bijection

$$
e: A^{\times} \backslash \operatorname{Hom}_{F}^{*}(K, A) \stackrel{\sim}{\longrightarrow} \mathcal{E}_{F}(K, A) .
$$

Proof. Let $\varphi$ and $\varphi^{\prime}$ be two maps in $\operatorname{Hom}_{F}^{*}(K, A)$, and let $V_{\varphi}$ and $V_{\varphi^{\prime}}$ be the induced $(K, \Delta)$-bimodule structures on $V$. Write

$$
V_{\varphi}=V_{1} \oplus \cdots \oplus V_{s} \quad \text { and } \quad V_{\varphi^{\prime}}=V_{1}^{\prime} \oplus \cdots \oplus V_{s}^{\prime},
$$


where $V_{i}$ and $V_{i}^{\prime}$ are $\left(K_{i}, \Delta\right)$-bimodules. We have shown (Lemma 2.5) that $\varphi$ and $\varphi^{\prime}$ are equivalent if and only if $V_{\varphi}$ and $V_{\varphi^{\prime}}$ are isomorphic as $(K, \Delta)$-bimodules, equivalently, $V_{i} \simeq V_{i}^{\prime}$ as $\left(K_{i}, \Delta\right)$-bimodules for $i=1, \ldots, s$. Since each $\Delta \otimes_{F} K_{i}$ is simple, the latter is the same as the condition $\operatorname{dim}_{\Delta} V_{i}=\operatorname{dim}_{\Delta} V_{i}^{\prime}$ for $i=1, \ldots, s$. One associates to $\varphi$ a tuple

$$
\mathbf{n}=\left(\operatorname{dim}_{\Delta} V_{1}, \ldots, \operatorname{dim}_{\Delta} V_{s}\right)
$$

which satisfies the condition (3.1) and determines the map $\varphi$ up to equivalence. As such tuples are in one-to-one correspondence with elements in $\mathcal{E}_{F}(K, A)$. Then we show a bijection map $e: A^{\times} \backslash \operatorname{Hom}_{F}^{*}(K, A) \rightarrow \mathcal{E}_{F}(K, A)$ which is given by

$$
e(\varphi)=\left(\operatorname{dim}_{\Delta} V_{1} / \ell_{1}, \ldots, \operatorname{dim}_{\Delta} V_{s} / \ell_{s}\right) .
$$

This completes the proof of the proposition.

3.2. Global results. In the remaining of this article let $F$ be a global field. Let $A$ be a central simple algebra over $F$ and $K$ a finite field extension over $F$. We use the following notations.

- $A=\operatorname{End}_{\Delta}(V)$, where $\Delta$ is the division part of $A$, and $V$ is a finite right $\Delta$-module of rank $n$.

- $k:=[K: F]$ and $\delta_{0}:=\operatorname{deg}(\Delta)$.

- For any place $v$ of $F$, denote by $F_{v}$ the completion of $F$ at $v$. Put

$$
K_{v}:=K \otimes F_{v}=\prod_{w \mid v} K_{w}, \quad A_{v}:=A \otimes F_{v}, \quad \Delta_{v}=\Delta \otimes F_{v}=\operatorname{Mat}_{s_{v}}\left(D_{v}\right),
$$

where $D_{v}$ is the division part of the central simple algebra $\Delta_{v}$ (we do not use the letter $D$ as an algebra over $F$ in this section; do not confuse $D_{v}$ as the completion of $D$ ) and $s_{v}$ is the capacity of $\Delta_{v}$.

- $k_{w}:=\left[K_{w}: F_{v}\right]$ and $d_{v}:=\operatorname{deg}\left(D_{v}\right)$, where $w$ is a place of $K$ over $v$.

- $\Delta \otimes_{F} K=\operatorname{Mat}_{c}\left(\Delta^{\prime}\right)$ and $\delta^{\prime}:=\operatorname{deg}\left(\Delta^{\prime}\right)$, where $\Delta^{\prime}$ is the division part of the central simple algebra $\Delta \otimes K$ over $K$, and $c$ is its capacity. One has

$$
\delta_{0}=\delta^{\prime} c .
$$

- For any place $w$ of $K$, put

$$
\Delta_{w}^{\prime}:=\Delta^{\prime} \otimes_{K} K_{w}=\operatorname{Mat}_{t_{w}}\left(D_{w}^{\prime}\right), \quad d_{w}^{\prime}:=\operatorname{deg}\left(D_{w}^{\prime}\right),
$$

where $D_{w}^{\prime}$ is the division part of the central simple algebra $\Delta_{w}^{\prime}$ and $t_{w}$ is the local capacity of $\Delta^{\prime}$ at $w$.

- $c_{w}:=\mathrm{c}\left(D_{v} \otimes_{F_{v}} K_{w}\right)$, i.e. $D_{v} \otimes_{F_{v}} K_{w}=\operatorname{Mat}_{c_{w}}\left(D_{w}^{\prime}\right)$. One has

$$
d_{v}=d_{w}^{\prime} c_{w}
$$

It follows from

$$
\begin{aligned}
& \Delta \otimes_{F} K_{w}=\left(\Delta \otimes F_{v}\right) \otimes_{F_{v}} K_{w}=\Delta_{v} \otimes K_{w}=\operatorname{Mat}_{s_{v} c_{w}}\left(D_{w}^{\prime}\right) \quad \text { and } \\
& \Delta \otimes_{F} K_{w}=\left(\Delta \otimes_{F} K\right) \otimes_{K} K_{w}=\operatorname{Mat}_{c}\left(\Delta^{\prime}\right) \otimes_{K} K_{w}=\operatorname{Mat}_{c t_{w}}\left(D_{w}^{\prime}\right)
\end{aligned}
$$

that

$$
s_{v} c_{w}=c t_{w} .
$$


- For any rational number $a \in \mathbb{Q}$, we write $\mathbf{d}(a)$ for the positive denominator of $a$ in its reduced form, and $\mathbf{n}(a)$ for its numerator.

- For each place $v$ of $F$, write

$$
\operatorname{inv}_{v}(\Delta)=\frac{a_{v}}{\delta_{0}}=\frac{a_{v}^{\prime} s_{v}}{d_{v} s_{v}}=\frac{a_{v}^{\prime}}{d_{v}}, \quad \operatorname{gcd}\left(a_{v}^{\prime}, d_{v}\right)=1 \text { and } s_{v}=\operatorname{gcd}\left(a_{v}, \delta_{0}\right) .
$$

One has, by the Grunwald-Wang theorem

$$
\delta_{0}=\operatorname{lcm}\left\{d_{v}\right\}_{v \in V^{F}} \quad \text { and } \quad\left(\operatorname{gcd}\left\{a_{v}\right\}_{v \in V^{F}}, \delta_{0}\right)=1,
$$

where $V^{F}$ denotes the set of all places of $F$.

- For each place $w$ of $K$, write

$$
\operatorname{inv}_{w}\left(\Delta^{\prime}\right)=\frac{b_{w}}{\delta^{\prime}}=\frac{b^{\prime}{ }_{w} t_{w}}{d_{w}^{\prime} t_{w}}=\frac{{b^{\prime}}_{w}}{d_{w}^{\prime}}, \quad \operatorname{gcd}\left(b^{\prime}{ }_{w}, d_{w}^{\prime}\right)=1 \text { and } t_{w}=\operatorname{gcd}\left(b_{w}, \delta^{\prime}\right) .
$$

One has

$$
\delta^{\prime}=\operatorname{lcm}\left\{d_{w}^{\prime}\right\}_{w \in V^{K}} \quad \text { and } \quad\left(\operatorname{gcd}\left\{b_{w}\right\}_{w \in V^{K}}, \delta^{\prime}\right)=1,
$$

where $V^{K}$ denotes the set of all places of $K$.

- It follows from $\operatorname{inv}\left(D_{w}^{\prime}\right)=\operatorname{inv}\left(D_{v}\right)\left[K_{w}: F_{v}\right]$ (see [13]) that

$$
c_{w}=\operatorname{gcd}\left(d_{v}, k_{w}\right)
$$

Given $K$ and $A$, we have, for each place $v$ of $F$,

- a tuple $\left(k_{w}\right)_{w \mid v}$ of positive integers, and

- a rational number $\operatorname{inv}_{v}(\Delta)=a_{v}^{\prime} / d_{v}$

satisfying the following conditions:

(a) $\sum_{w \mid v} k_{w}=k$ for all $v \in V^{F}$,

(b) (i) $d_{v}=1$ if $v$ is a complex place,

(ii) $d_{v} \in\{1,2\}$ if $v$ is a real place,

(iii) $d_{v}=1$ for almost all $v$, and

(iv) (Global class field theory) one has

$$
\sum_{v \in V^{F}} \frac{a^{\prime}{ }_{v}}{d_{v}}=0 \quad(\text { in } \mathbb{Q} / \mathbb{Z}) .
$$

We compute all other numerical invariants $\delta_{0}, c_{w}, d_{w}^{\prime}, \delta^{\prime}$ and $c$ as follows.

(i) The (global) degree $\delta_{0}$ of $\Delta$ can be computed by (3.11).

(ii) Then one computes the local capacity $c_{w}$ of $D_{v} \otimes_{F_{v}} K_{w}$ and the (local) degree $d_{w}^{\prime}$ of $D_{w}^{\prime}$ by (3.13) and (3.9), respectively.

(iii) Using (3.12) we compute the (global) degree $\delta^{\prime}$ of $\Delta^{\prime}$ and then compute the (global) capacity $c$ of $\Delta \otimes K$ using (3.8).

We define the following condition (G stands for "global")

(G) $k \mid n c$. 
Proposition 3.3. The set $\operatorname{Hom}_{F}(K, A)$ is non-empty if and only if the condition (G) holds.

Proof. This follows from Lemma 2.3.

Now we formulate the corresponding local conditions. Note that

$$
K_{v}=\prod_{w \mid v} K_{w} \quad \text { and } \quad A_{v}=\operatorname{Mat}_{n s_{v}}\left(D_{v}\right) .
$$

Put

$$
\mathcal{E}:=A^{\times} \backslash \operatorname{Hom}_{F}(K, A)
$$

the Noether-Skolem theorem says that if this set is non-empty then it has one element. For each place $v$ of $F$, define a set (c.f. (3.5))

$$
\mathcal{E}_{v}:=\mathcal{E}_{F_{v}}\left(K_{v}, A_{v}\right)=\left\{\left(x_{w}\right)_{w \mid v} \mid x_{w} \in \mathbb{N}, \sum_{w \mid v} \ell_{w} x_{w}=n s_{v}\right\},
$$

where $\ell_{w}:=k_{w} / c_{w}$. Define the following condition (L stands for "local")

(L) The set $\mathcal{E}_{v}$ is non-empty for all $v \in V^{F}$.

Proposition 3.4. There is an embedding of $K_{v}$ in $A_{v}$ over $F_{v}$ if and only if the set $\mathcal{E}_{v}$ is non-empty.

Proof. This follows from Proposition 3.2.

We have the following implication

the condition $(\mathbf{G})$ holds $\Longrightarrow$ the condition (L) holds.

The local-global principle then asks whether the converse is also true.

\subsection{Special vectors and the local-global principle. Let}

$$
e_{v}: A_{v}^{\times} \backslash \operatorname{Hom}_{F_{v}}^{*}\left(K_{v}, A_{v}\right) \stackrel{\sim}{\longrightarrow} \mathcal{E}_{v}
$$

be the corresponding bijection obtained in Proposition 3.2. Let us suppose first that the set $\operatorname{Hom}_{F}(K, A)=\operatorname{Hom}_{F}^{*}(K, A)$ of embeddings of $K$ into $A$ over $F$ is non-empty. For any element $\varphi$ in $\operatorname{Hom}_{F}(K, A)$, let $\varphi_{v} \in \operatorname{Hom}_{F_{v}}\left(K_{v}, A_{v}\right)$ be the extension of $\varphi$ by $F_{v}$-linearity, and let $\left[\varphi_{v}\right]$ be its equivalence class. Then one defines an element $\mathbf{x}_{v} \in \mathcal{E}_{v}$ by

$$
\mathbf{x}_{v}:=e_{v}\left(\left[\varphi_{v}\right]\right) .
$$

The association $\varphi \mapsto \mathbf{x}_{v}$ induces a well-defined map, which we denote again by $e_{v}$,

$$
e_{v}: \mathcal{E} \rightarrow \mathcal{E}_{v}
$$

The non-emptiness of $\operatorname{Hom}_{F}(K, A)$ implies the existence of such a vector $\mathbf{x}_{v}$ in $\mathcal{E}_{v}$ for each place $v \in V^{F}$. We now calculate these special vectors explicitly.

The map $\varphi$ gives rise to a $(K, \Delta)$-bimodule structure on $V$. Since $V$ is free $K$ module of rank $n \delta_{0}^{2} / k$, its completion $V \otimes_{F} F_{v}$ is also a free $K_{v}$-module of same rank. Therefore, one has the decomposition

$$
V \otimes F_{v}=\bigoplus_{w \mid v} V_{w}
$$


where each factor $V_{w}$ is a $\left(K_{w}, \operatorname{Mat}_{s_{v}}\left(D_{v}\right)\right)$-bimodule of $K_{w}$-rank $n \delta_{0}^{2} / k$ (recall that $\left.\Delta_{v}=\operatorname{Mat}_{s_{v}}\left(D_{v}\right)\right)$. Using the Morita equivalence, the module $V_{w}$ is isomorphic to $W_{w}^{\oplus s_{v}}$ for a $\left(K_{w}, D_{v}\right)$-bimodule $W_{w}$ of $D_{v}$-rank $n s_{v} k_{w} / k$. Using the formula (3.7), the $w$-component $\mathbf{x}_{w}$ of the vector $\mathbf{x}_{v}$ is given by

$$
\mathbf{x}_{w}:=\operatorname{dim}_{D_{v}} W_{w} / \ell_{w}=n s_{v} c_{w} / k
$$

which is a positive integer. Recall that $c_{w}=\operatorname{gcd}\left(k_{w}, d_{v}\right)$ and $\ell_{w}=k_{w} / c_{w}$.

Therefore, this leads us to the following definition of special vectors no matter the set $\mathcal{E}$ is non-empty or not. For each place $v$ of $F$ we define a vector (still denoted by) $\mathbf{x}_{v}=\left(\mathbf{x}_{w}\right)_{w \mid v} \in \prod_{w \mid v} \mathbb{Q}_{>0}$ by (3.16), and we call them special vectors. The above calculation shows if the set $\mathcal{E}$ is non-empty, then the vector $\mathbf{x}_{v}$ is the image of the $\operatorname{map} e_{v}$.

Proposition 3.5. Notations as above. If the set $\mathcal{E}$ is non-empty, then one has

$$
\mathbf{x}_{v} \in \mathcal{E}_{v}, \quad \forall v \in V^{F},
$$

or equivalently, each vector $\mathbf{x}_{v}$ lies in $\prod_{w \mid v} \mathbb{N}$ for all $v \in V^{F}$.

If we denote by $\overline{\mathbf{x}}_{w}$ the class of $\mathbf{x}_{w}$ in $\mathbb{Q} / \mathbb{Z}$, then we associate to the pair $(K, A)$ an element

$$
\overline{\mathbf{x}}=\left(\overline{\mathbf{x}}_{w}\right)_{w \in V^{K}} \in \bigoplus_{w \in V^{K}} \mathbb{Q} / \mathbb{Z}
$$

Then Proposition 3.5 states that the vanishing of the class $\overline{\mathbf{x}}$ is a necessary condition for the set $\mathcal{E}$ to be non-empty. The following result states that this is the only obstruction to the local-global principle.

TheOREM 3.6. Notations as above. We have

$$
\operatorname{Hom}_{F}^{*}(K, A) \neq \emptyset \Longleftrightarrow \overline{\mathbf{x}}=0 \text {. }
$$

Proof. Note that the condition $\overline{\mathbf{x}}=0$ implies $\mathbf{x}_{v} \in \mathcal{E}_{v}$ and hence $\operatorname{Hom}_{F_{v}}^{*}\left(K_{v}, A_{v}\right)$ is non-empty for all $v \in V^{F}$. The implication $\Longrightarrow$ is already proved. To show the other direction, we must show that the condition $(\mathbf{G}) k \mid n c$ holds. Using $c=\delta_{0} / \delta^{\prime}$ and $\delta^{\prime}=\operatorname{lcm}\left\{d_{w}^{\prime}\right\}$, we rewrite the condition $(\mathbf{G})$ as

$$
k \mid\left(n \delta_{0} / d_{w}^{\prime}\right), \quad \forall w \in V^{K}
$$

Using (3.9) and (3.16), we have

$$
\mathbf{x}_{w}=n s_{v} c_{w} / k=n s_{v} d_{v} / k d_{w}^{\prime}=n \delta_{0} / k d_{w}^{\prime} \in \mathbb{N}
$$

for all $w \in V^{K}$. This verifies the condition $(\mathbf{G})$ and hence proves the theorem.

3.4. An example. We will show an example of a pair $(K, A)$, where $K / F$ is a Galois extension and $A$ is a central simple $F$-algebra so that

- the set $\operatorname{Hom}_{F_{v}}^{*}\left(K_{v}, A_{v}\right)$ is non-empty for all $v \in V^{F}$, and

- the set $\operatorname{Hom}_{F}(K, A)$ is empty. 
This particularly shows that the question (Q3) has a negative answer. Let $K / F$ be a Galois extension of degree 8. Choose two places $v_{1}$ and $v_{2}$ of $F$ so that $k_{w}=2$ for all $w \mid v_{1}$ or $w \mid v_{2}$. Such places exist by the Chebotarev density theorem. Let $A$ be a central simple algebra over $F$ of degree 24 that is ramified exactly at the two places $v_{1}$ and $v_{2}$, and

$$
A_{v_{1}}=\operatorname{Mat}_{6}\left(D_{v_{1}}\right), \text { and } A_{v_{2}}=\operatorname{Mat}_{6}\left(D_{v_{2}}\right)
$$

where $D_{v_{1}}$ and $D_{v_{2}}$ are central division algebras of degree 4 . The existence of such an $A$ follows from the global class field theory. One has $c_{w}=2$ and $\ell_{w}=1$. The sets $\mathcal{E}_{v}$ for unramified places $v$ are non-empty. For $v=v_{i}, i=1,2$, one see that

$$
\mathcal{E}_{v_{i}}=\left\{\left(x_{i}\right) \in \mathbb{N}^{4} \mid \sum_{i=1}^{4} x_{i}=6\right\} \neq \emptyset .
$$

By Proposition 3.4, the sets $\operatorname{Hom}_{F_{v}}^{*}\left(K_{v}, A_{v}\right)$ are non-empty for all $v \in V^{F}$. On the other hand, $\mathbf{x}_{w}=6 c_{w} / k=12 / 8$ for any $w \mid v_{1}$ or $w \mid v_{2}$, which is not an integer. By Theorem 3.6, the set $\operatorname{Hom}_{F}(K, A)$ is empty.

The same argument shows that there is a Galois extension $K / F$ of degree $p^{m}$, and a central simple algebra $A / F$ of degree $p^{m} q$, where $p$ and $q$ are primes, with $m \geq 2$ and $p<q$, so that the local-global principle for embedding $K$ into $A$ fails. One takes $k_{w}=p$ and $d_{v}=p^{2}$. Then $c_{w}=p$ and $\ell_{w}=1$, We see that $\mathcal{E}_{v_{i}}$ is non-empty from the inequality $p^{m-1} \leq p^{m-2} q$. However, $\mathbf{x}_{w}=p^{m-2} q p / p^{m} \notin \mathbb{N}$.

4. The local-global principle. Keep the notation in $\S 3.2$ and 3.3 . In this section, we study on the local-global principle in detail.

For the convenience of discussion, we make the following definitions.

DEFINITION 4.1 .

(1) Let $K$ be a finite field extension over a global field $F$ and $A$ be a central simple algebra over $F$. We say that the Hasse principle for the pair $(K, A)$ holds if the following properties are equivalent:

$$
\operatorname{Hom}_{F_{v}}^{*}\left(K_{v}, A_{v}\right) \neq \emptyset, \quad \forall v \in V^{F} \Longleftrightarrow \operatorname{Hom}_{F}^{*}(K, A)=\operatorname{Hom}_{F}(K, A) \neq \emptyset .
$$

In other words, the conditions $(\mathbf{G})$ and $(\mathbf{L})$ in $\S 3.2$ are equivalent.

(2) Let $F$ be a global field. Let $(k, \delta)$ be a pair of two positive integers with $k \mid \delta$. We say that the Hasse principle for the pair $(k, \delta)$ holds if for any finite field extension $K$ over $F$ of degree $k$ and for any central simple algebra $A$ over $F$ of degree $\operatorname{deg}(A)=\delta$, the Hasse principle for the pair $(K, A)$ holds.

Theorem 3.6 gives us an effective way to check the Hasse principle. Namely, one only needs to check

$$
\mathbf{x}_{w}=n s_{v} c_{w} / k \stackrel{?}{\in} \mathbb{N}, \quad \forall w \in V^{K}
$$

for a given pair $(K, A)$.

\subsection{Basic positive results.}

Proposition 4.2. Let $A$ be a central simple algebra over $F$ with index $\mathrm{i}(A)=\delta_{0}$ and degree $\operatorname{deg} A=n \delta_{0}$, and let $K$ be a finite field extension over $F$ of degree $[K$ : $F] \mid \operatorname{deg} A$. Suppose one of the following properties holds: 
(1) $[K: F]=\operatorname{deg} A$.

(2) $K$ splits $A$.

(3) For any place $v$, the algebra $A_{v}$ is either a division algebra or a matrix algebra over $F_{v}$.

Then the Hasse principle for the pair $(K, A)$ holds.

Proof.

(1) This is a well-known result; see Pierce $[8, \S 18.4]$ or Prasad-Rapinchuk [9, Proposition A.1].

(2) Since $K$ splits $A$, we have $d_{w}^{\prime}=1$ for all $w \in V^{K}$ and hence $\delta^{\prime}=\underset{w}{\operatorname{lcm}}\left\{d_{w}^{\prime}\right\}=1$ and $c=\delta_{0}$. Thus,

$$
k\left|n \delta_{0} \Longleftrightarrow k\right| n c .
$$

This verifies the condition $(\mathbf{G})$ and hence $\operatorname{Hom}_{F}(K, A) \neq \emptyset$.

(3) Suppose there exists an embedding of $K_{v}$ into $A_{v}$ for all $v \in V^{F}$. If $A_{v}$ is a central division algebra, then $K_{v}=K_{w}$ is a field extension over $F_{v}$ with $\left[K_{w}: F_{v}\right]=k$ and $c_{w}=k$. Then we have

$$
\mathbf{x}_{w}=n s_{v} c_{w} / k=n \in \mathbb{N}, \quad \forall w \mid v .
$$

For the other case that $A_{v}$ is a matrix algebra over $F_{v}$, we have $s_{v}=\delta_{0}$. Thus, by the assumption $k \mid n \delta_{0}$, we have

$$
\mathbf{x}_{w}=n s_{v} c_{w} / k=n \delta_{0} c_{w} / k \in \mathbb{N}, \quad \forall w \mid v .
$$

By Theorem 3.6, the set $\operatorname{Hom}_{F}(K, A)$ is not empty.

4.2. Construction of counterexamples. Let $K$ be a finite field extension over $F$ with $[K: F]=k=p_{1}^{m_{1}} \cdots p_{r}^{m_{r}}$, and $A$ be a central division algebra over $F$ of degree $\delta=p_{1}^{n_{1}} \cdots p_{r}^{n_{r}}$, where $p_{i}$ is a prime number, $n_{i} \in \mathbb{N}$, and $m_{i} \in \mathbb{Z}_{\geq 0}$ with $m_{i} \leq n_{i}$ for $i=1, \ldots, r$.

We shall construct examples for which the Hasse principle does not hold. Recall (Definition 4.1) that the Hasse principle for a pair $(k, \delta)$ does not hold if there exists a pair $(K, A)$ such that $\operatorname{Hom}_{F_{v}}^{*}\left(K_{v}, A_{v}\right) \neq \emptyset$ for all $v \in V^{F}$ but $\operatorname{Hom}_{F}(K, A)=\emptyset$.

The construction is to set local data $s_{v}, d_{v}$, and a partition $k=\sum_{w \mid v} k_{w}$ of the integer $k$ for some place $v$ of $F$ so that some $w$-component $\mathbf{x}_{w}$ of the special vector $\mathbf{x}_{v}$ is not integral. Then we apply Theorem 3.6 to conclude that the Hasse principle for certain pair $(K, A)$ does not hold. To ensure that the data $k=\sum_{w \mid v} k_{w}$ come from a global field $K$, we need the following result.

Lemma 4.3 (c.f. [16, Lemma 3.2]). Let $S$ be a finite subset of $V^{F}$. Let $L_{v}$, for each $v \in S$, be any etale algebra over $F_{v}$ of same degree $\left[L_{v}: F_{v}\right]=n$. Then there is a finite separable field extension $K$ over $F$ of degree $n$ such that $K \otimes_{F} F_{v} \simeq L_{v}$ for all $v \in S$.

Moreover, one also needs to ensure that the data $s_{v}$ and $d_{v}$ come from a central division algebra $A$ over $F$. For this, we need the global class field theory; see [12, § 32] or [4].

THEOREM 4.4. Let $S$ be a finite subset of $V^{F}$. For any positive integer $\delta$, suppose we are given any set of rational numbers $\left\{a_{v} / d_{v}\right\}_{v \in S}$ with $\operatorname{gcd}\left(a_{v}, d_{v}\right)=1$ such that 
(1) $\operatorname{lcm}_{v \in S}\left\{d_{v}\right\}=\delta$.

(2) $\sum_{v \in S}^{v \in S} a_{v} / d_{v}=0 \in \mathbb{Q} / \mathbb{Z}$.

(3) $d_{v}=1$ if $v$ is complex.

(4) $d_{v}=1$ or 2 if $v$ is real.

Then up to isomorphism, there is a unique central division algebra $A$ over $F$ with $\operatorname{deg} A=\delta$ such that $\operatorname{inv} A_{v}=a_{v} / d_{v} \in \mathbb{Q} / \mathbb{Z}$ for all $v \in S$ and inv $A_{v}=0 \in \mathbb{Q} / \mathbb{Z}$ for all $v \notin S$.

Let $(k, \delta)$ be a pair of positive integers with $k \mid \delta$. For any partition $\lambda$ of $k$

$$
\lambda=\left(k_{1}, \ldots, k_{t}\right), \quad k_{1} \leq \cdots \leq k_{t}, \quad \sum_{i=1}^{t} k_{i}=k
$$

and a positive integer $s$ dividing $\delta$, we set

$$
\mathcal{E}(\lambda, s):=\left\{\left(x_{i}\right) \in \mathbb{N}^{t} \mid \sum_{i=1}^{t} \ell_{i} x_{i}=s\right\},
$$

where $\ell_{i}:=k_{i} / \operatorname{gcd}\left(k_{i}, d\right)$, and define a vector $\mathbf{x}(\lambda, s) \in \mathbb{Q}^{t}$, where

$$
\mathbf{x}(\lambda, s):=\left(\mathbf{x}_{i}\right), \quad \mathbf{x}_{i}=\mathbf{x}_{i}(\lambda, s):=s \operatorname{gcd}\left(k_{i}, d\right) / k, \quad \forall i=1 \ldots, t .
$$

Let $\operatorname{LD}(k, \delta)$ be the set of pairs $(\lambda, s)$ of partitions $\lambda$ of $k$ and divisors $s$ of $\delta$ such that $\mathcal{E}(\lambda, s)$ is non-empty ("LD" stands for all possible local decompositions).

We transform the problem of checking the Hasse principle for the pair $(k, \delta)$ in purely combinatorial terms.

TheOREM 4.5. Let $F$ be a global field and let $(k, \delta)$ be a pair of positive integers with $k \mid \delta$.

(1) Suppose for all elements $(\lambda, s) \in \operatorname{LD}(k, \delta)$, the vector $\mathbf{x}(\lambda, s)$ has integral components. Then the Hasse principle for $(k, \delta)$ holds.

(2) Suppose there is an element $(\lambda, s) \in \mathrm{LD}(k, \delta)$ such that $\mathbf{x}_{i}(\lambda, s) \notin \mathbb{N}$ for some $i$. Then there are a finite separable field extension $K$ of degree $k$ over $F$ and a central division algebra $A$ of degree $\delta$ over $F$ so that the Hasse principle for $(K, A)$ fails. In particular, the Hasse principle for the pair $(k, \delta)$ fails.

Proof. (1) Let $K$ be any finite field extension of $F$ of degree $k$ and $A$ be any central simple algebra over $F$ of degree $\delta$. For any place $v$ of $F$, we get a partition $\lambda=\left(k_{w}\right)_{w \mid v}$ of $k$ and a positive divisor $s$ of $\delta$ as the capacity of $A_{v}=A \otimes F_{v}$. The assumption $\operatorname{Hom}_{F_{v}}^{*}\left(K_{v}, A_{v}\right) \neq \emptyset$ assures that $\mathcal{E}(\lambda, s)=\mathcal{E}_{v}$ is non-empty. Therefore, the pair $(\lambda, s)$ is an element in $\operatorname{LD}(k, \delta)$. Then the assumption gives that $\mathbf{x}_{v}=\mathbf{x}(\lambda, s)$ has integral components. This works for all $v \in V^{F}$. By Theorem 3.6, we have $\operatorname{Hom}_{F}^{*}(K, A) \neq \emptyset$.

(2) Let $(\lambda, s) \in \operatorname{LD}(k, \delta)$, where $\lambda=\left(k_{1}, \ldots, k_{t}\right)$ and $s \mid \delta$, be an element such that $\mathbf{x}(\lambda, s)$ is not an integral vector. Choose any finite set $S=\left\{v_{1}, v_{1}^{\prime}, v_{2}, v_{2}^{\prime}\right\}$ of 4 finite places of $F$. Let $A$ be the central division algebra over $F$ of degree $\delta$ with following local invariants (Theorem 4.4):

- $\operatorname{inv}_{v_{1}}(A)=-\operatorname{inv}_{v_{1}^{\prime}}(A)=1 / d$ and $\operatorname{inv}_{v_{2}}(A)=-\operatorname{inv}_{v_{2}^{\prime}}(A)=1 / \delta$.

- $\operatorname{inv}_{v}(A)=0$ for all $v \notin S$. 
Let $L_{v_{2}}$ and $L_{v_{2}^{\prime}}$ be any finite separable field extensions of degree $k$ over $F_{v_{2}}$ and $F_{v_{2}^{\prime}}$, respectively. Let $L_{v_{1}}=\prod_{i=1}^{t} E_{i}$ and $L_{v_{1}^{\prime}}=\prod_{i=1}^{t} E_{i}^{\prime}$ where $E_{i}$ (resp. $E_{i}^{\prime}$ ) is a separable field extension of $F_{v_{1}}$ (resp. of $F_{v_{1}^{\prime}}$ ) of degree $k_{i}$ for all $i$. By Lemma 4.3, there exists a finite separable field extension $K$ of $F$ of degree $k$ such that $K \otimes F_{v} \simeq L_{v}$ for all $v \in S$. It follows from $(\lambda, s) \in \operatorname{LD}(k, \delta)$ that $\mathcal{E}_{v_{1}}$ and $\mathcal{E}_{v_{1}^{\prime}}$ are non-empty. Also the sets $\mathcal{E}_{v_{2}}$ and $\mathcal{E}_{v_{2}^{\prime}}$ are non-empty as $K_{v_{2}}$ and $K_{v_{2}^{\prime}}$ are fields. Since $A_{v}$ is a matrix algebra for $v \notin S$, these sets $\mathcal{E}_{v}$ are non-empty, too. Finally, since $\mathbf{x}(\lambda, s)$ is not integral, the special vector $\mathbf{x}_{v_{1}}=\mathbf{x}(\lambda, s)$ is not integral. Therefore, we have constructed a pair $(K, A)$ for which the Hasse principle fails.

Proposition 4.6. Let $\delta=p_{1}^{n_{1}} \cdots p_{r}^{n_{r}}$, and let $k$ be a positive integer with $k>1$ and $k \mid \delta$, where $p_{i}^{\prime}$ s are prime divisors of $\delta$. Assume that $k \leq \delta / p_{i}^{n_{i}}$ and $p_{i} \mid k$ for some $1 \leq i \leq r$ (so $r \geq 2$ ). Then the Hasse principle for the pair $(k, \delta)$ does not hold.

Proof. Without loss of generality, we may assume $i=1$. We write $k=p_{1}^{m_{1}} k^{\prime}$ for some prime-to- $p_{1}$ integer $k^{\prime}$. Let

(i) $s=\delta / p_{1}^{n_{1}}=p_{2}^{n_{2}} \cdots p_{r}^{n_{r}}$.

(ii) $\lambda=(1, \ldots, 1)=:\left(1^{\oplus k}\right)$.

Then one has

$$
d=p_{1}^{n_{1}}, \quad c_{i}:=\operatorname{gcd}\left(k_{i}, d\right)=1, \quad \ell_{i}:=k_{i} / c_{i}=1,
$$

and

$$
\mathbf{x}_{i}=s / k=p_{2}^{n_{2}} \cdots p_{r}^{n_{r}} / p_{1}^{m_{1}} k^{\prime} \notin \mathbb{N}, \quad \forall i
$$

Note that the condition $k \leq \delta / p_{1}^{n_{1}}=s$ implies the equation $X_{1}+\cdots+X_{k}=s$ has a positive integral solution, i.e. the set $\mathcal{E}(\lambda, s)$ is non-empty. By Theorem 4.5 , the Hasse principle does not hold.

Corollary 4.7. Let $\delta$ be a positive integer divisible by at least two primes. Then there is a positive integer $k$ with $k \mid \delta$ such that the Hasse principle for the pair $(k, \delta)$ does not hold.

Proof. Write $\delta=p_{1}^{n_{1}} \cdots p_{r}^{n_{r}}, r \geq 2$, and assume that $p_{1}$ is the smallest prime divisor. Let $k=p_{1}$. Then the corollary follows from Proposition 4.6.

COROllary 4.8. Let $\delta$ be a positive integer divisible by at least two primes. Then there are a central simple algebra $A$ of degree $\delta$ and a finite field extension of $F$ of degree $[K: F] \mid \delta$ so that the Hasse principle for the pair $(K, A)$ does not hold.

5. Hasse principle for homogeneous spaces. The space of all embeddings of an etale commutative algebra $K$ into $A$ over $F$ forms an variety $X$ over $F$. It is naturally equipped with an action by the algebraic group $A^{\times}$through conjugation. Therefore, the space $X$ is decomposed into several orbits and each orbit is a homogeneous space of $A^{\times}$. In the previous section, we see some counterexamples for the Hasse principle in a more combinatorial way. A closer look at these counterexamples would find out that some of local points actually lie in different orbits. This is the main reason that causes the failure of the Hasse principle. It turns out this is the only reason that fails the Hasse principle. Namely, if we requires that all local points lie in the same orbit $Y$, then $Y$ indeed has an $F$-rational point. This the main result of this section; see Theorem 5.4. 
5.1. F-kernels and $H^{2}$. We recall the definition and basic properties of the second non-abelian Galois cohomology $H^{2}$. Our references are Springer [14] and Borovoi [1].

Let $F$ be a field and let $F^{\text {sep }}$ denote a separable closure of $F$. Put $\Gamma_{F}$ : $\operatorname{Gal}\left(F^{\text {sep }} / F\right)$. Let $\bar{G}$ be a linear algebraic group over $F^{\text {sep }}$ and let $p: \bar{G} \rightarrow \operatorname{Spec} F^{\text {sep }}$ be the structure morphism.

For $\sigma \in \Gamma_{F}$, let $\sigma_{\natural}:$ Spec $F^{\text {sep }} \rightarrow \operatorname{Spec} F^{\text {sep }}$ denote the isomorphism induced by the map $\sigma^{-1}$. We have $(\sigma \tau)_{\natural}=\sigma_{\natural} \tau_{\natural}$ for $\sigma, \tau \in \Gamma_{F}$.

Let $\sigma \in \Gamma_{F}$. We say that an automorphism of schemes $s: \bar{G} \rightarrow \bar{G}$ is a $\sigma$ semialgebraic automorphism of $\bar{G}$ if the following diagram

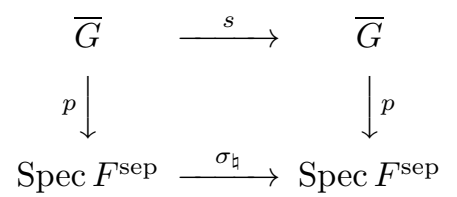

commutes and the induced morphism $s^{\prime}: \bar{G} \rightarrow \bar{G}^{\sigma}$ is an isomorphism of algebraic groups over Spec $F^{\text {sep }}$, where $\bar{G}^{\sigma}:=\bar{G} \times_{\sigma_{\natural}}$ Spec $F^{\text {sep }}$ is the base change deduced by the morphism $\sigma_{b}$. An semialgebraic automorphism $s$ of $\bar{G}$ is a $\sigma$-semialgebraic automorphism of $\bar{G}$ for some $\sigma \in \Gamma_{F}$. Define $\gamma(s):=\sigma$ if $s$ is a $\sigma$-semialgebraic automorphism of $\bar{G}$.

Let SAut $\bar{G}$ denote the group of semialgebraic automorphisms of $\bar{G}$. The map $\gamma:$ SAut $\bar{G} \rightarrow \Gamma_{F}$ is a homomorphism. Let Aut $\bar{G}$ denote the group of algebraic automorphisms of $\bar{G}$ over $F^{\text {sep }}$. We have an embedding Aut $\bar{G} \rightarrow$ SAut $\bar{G}$ and an exact sequence

$$
1 \longrightarrow \operatorname{Aut} \bar{G} \longrightarrow \text { SAut } \bar{G} \stackrel{\gamma}{\longrightarrow} \Gamma_{F} .
$$

Let Int $\bar{G}$ denote the group of inner automorphisms of $\bar{G}$. Set Out $\bar{G}:=\operatorname{Aut} \bar{G} / \operatorname{Int} \bar{G}$ and SOut $\bar{G}:=$ SAut $\bar{G} / \operatorname{Int} \bar{G}$. We have an exact sequence

$$
1 \longrightarrow \text { Out } \bar{G} \longrightarrow \text { SOut } \bar{G} \stackrel{q}{\longrightarrow} \Gamma_{F} \text {. }
$$

Definition 5.1. An F-kernel is a pair $L=(\bar{G}, \kappa)$, where $\bar{G}$ is an $F^{\text {sep-group }}$ and $\kappa: \Gamma_{F} \rightarrow$ SOut $\bar{G}$ is a homomorphism satisfying the following two conditions:

(a) $\kappa$ is a splitting of (5.1);

(b) $\kappa$ can be lifted to a continuous map $f: \Gamma_{F} \rightarrow$ SAut $\bar{G}$; here "continuous" means that the stabilizer in $\Gamma_{F}$ of any regular function $\phi \in F^{\operatorname{sep}}[\bar{G}]$ is open.

For an $F^{\text {sep }}$-kernel $L=(\bar{G}, \kappa)$, the second Galois cohomology set $H^{2}(F, L)=$ $H^{2}(F, \bar{G}, \kappa)$ is defined as follows (also see [1, p. 221]). A 2-cocycle is a pair $(f, u)$ of continuous maps

$$
f: \Gamma_{F} \rightarrow \operatorname{SAut} \bar{G}, \quad u: \Gamma_{F} \times \Gamma_{F} \rightarrow \bar{G}\left(F^{\mathrm{sep}}\right)
$$

such that for any $\sigma, \tau, v \in \Gamma_{F}$, the following holds:

$$
\begin{aligned}
\operatorname{int}\left(u_{\sigma, \tau}\right) \circ f_{\sigma} \circ f_{\tau} & =f_{\sigma \tau}, \\
u_{\sigma, \tau v} \cdot f_{\sigma}\left(u_{\tau, v}\right) & =u_{\sigma \tau, v} \cdot u_{\sigma, \tau}, \\
f_{\sigma} \bmod \operatorname{Int} \bar{G} & =\kappa(\sigma) .
\end{aligned}
$$


Let $Z^{2}(F, L)$ denote the set of 2-cocycles with coefficients in $L$. The group $C(F, \bar{G})$ of continuous maps $c: \Gamma_{F} \rightarrow \bar{G}\left(F^{\mathrm{sep}}\right)$ acts on $Z^{2}(F, L)$ on the left by

$$
c \cdot(f, u)=\left(f^{\prime}, u^{\prime}\right)
$$

where

$$
f_{\sigma}^{\prime}=\operatorname{int}\left(c_{\sigma}\right) \circ f_{\sigma}, \quad u_{\sigma, \tau}^{\prime}=c_{\sigma \tau} \cdot u_{\sigma, \tau} \cdot f_{\sigma}\left(c_{\tau}\right)^{-1} \cdot c_{\sigma}^{-1}
$$

The quotient set $H^{2}(F, L):=C(F, \bar{G}) \backslash Z^{2}(F, L)$ is called the second Galois cohomology set of $F$ with coefficients in $L$. If $(f, u) \in Z^{2}(F, L)$, we write $\mathrm{Cl}(f, u)$ for the cohomology class of $(f, u)$ in $H^{2}(F, L)$. A neutral 2-cocycle is a cocycle of the form $(f, 1)$. A neutral cohomological class in $H^{2}(F, L)$ is the class of a neutral cocycle.

Let $G$ be an $F$-group. Set $\bar{G}:=G \otimes_{F} F^{\text {sep }}$. For $\sigma \in \Gamma_{F}$, let $\sigma_{*}: \bar{G}=\bar{G}^{\sigma} \rightarrow \bar{G}$ be the morphism induced by the fiber product

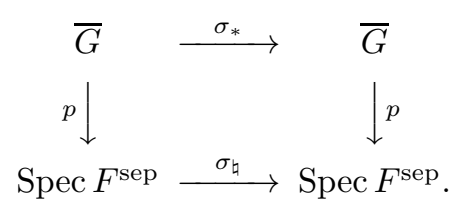

Then $\sigma_{*}$ is a $\sigma$-semialgebraic automorphism of $\bar{G}$. We have $(\sigma \tau)_{*}=\sigma_{*} \tau_{*}$ for $\sigma, \tau \in \Gamma_{F}$. We obtain a continuous homomorphism

$$
f_{G}: \Gamma_{F} \rightarrow \operatorname{SAut} \bar{G}, \quad \sigma \mapsto \sigma_{*}
$$

which splits the exact sequence (5.1). Composing $f_{G}$ with the homomorphism SAut $\bar{G} \rightarrow$ SOut $\bar{G}$, we obtain a homomorphism

$$
\kappa_{G}: \Gamma_{F} \rightarrow \operatorname{SOut} \bar{G}, \quad \kappa_{G}(\sigma)=\sigma_{*} \bmod \operatorname{Int} \bar{G}
$$

and, thus, an $F$-kernel $L_{G}=\left(\bar{G}, \kappa_{G}\right)$. Set $H^{2}(F, G):=H^{2}\left(F, L_{G}\right)$. When $G$ is commutative, $H^{2}(F, G)$ is the group cohomology $H^{2}\left(\Gamma_{F}, G\left(F^{\mathrm{sep}}\right)\right)$ and it is an abelian group. In $H^{2}(F, G)$, we have a neutral class $\mathrm{Cl}\left(f_{G}, 1\right)$, which we denote by $n(G)$.

Suppose $H^{2}(F, L)$ of an $F$-kernel $L=(\bar{G}, \kappa)$ contains a neutral class $\mathrm{Cl}(f, 1)$. Then by Weil's descent theorem there is an $F$-form $G$ of $\bar{G}$, unique up to $F$ isomorphism, such that $f=f_{G}$ and $\kappa=\kappa_{G}$. Let

$$
\psi: \Gamma_{F} \rightarrow(G / Z)\left(F^{\mathrm{sep}}\right)=\operatorname{Int} \bar{G}
$$

be a cocycle, where $Z$ is the center of $G$. The map $\psi$ defines an inner form $G^{\prime}={ }_{\psi} G$ of $G$ and gives rise to a homomorphism $f^{\prime}:=f_{G^{\prime}}$. We have $f_{\sigma}^{\prime}=\psi_{\sigma} f_{\sigma}$ for all $\sigma \in \Gamma_{F}$ and $\kappa_{G^{\prime}}=\kappa$. This defines another neutral class $n\left(G^{\prime}\right)=\mathrm{Cl}\left(f^{\prime}, 1\right)$ in $H^{2}(F, L)$. Conversely, any neutral class arises in this way. Indeed, one puts $\psi_{\sigma}:=f_{\sigma}^{\prime} f_{\sigma}^{-1}$ and then the map $\psi: \Gamma_{F} \rightarrow \operatorname{Int} \bar{G}$ is a cocycle.

5.2. Homogeneous spaces. Let $H$ be a connected reductive group over $F$. Let $X$ be a right homogeneous space of $H$. The homogeneous space $X$ gives rise to an $F$-kernel $L$ as follows. Let $x \in X\left(F^{\mathrm{sep}}\right)$ be an $F^{\mathrm{sep}}$-point and let $\bar{G}$ be the stabilizer subgroup of $x$ in $\bar{H}:=H \otimes_{F} F^{\text {sep }}$. For $\sigma \in \Gamma_{F}$, write

$$
{ }^{\sigma} x=x \cdot h_{\sigma}
$$


where $h_{\sigma} \in H\left(F^{\text {sep }}\right)$. As $\Gamma_{F}$ acts continuously on the discrete set $X\left(F^{\text {sep }}\right)$, we have a continuous map $h: \Gamma_{F} \rightarrow H\left(F^{\mathrm{sep}}\right)$. The $\sigma$-semialgebraic automorphism of $\bar{H}$

$$
f_{\sigma}:=\operatorname{int}\left(h_{\sigma}\right) \circ \sigma_{*}
$$

takes $\bar{G}$ into itself. To see this, if $g \in \bar{G}$, then we have $x \cdot g=x$ and ${ }^{\sigma} x \cdot{ }^{\sigma} g={ }^{\sigma} x$. So $x \cdot h_{\sigma}{ }^{\sigma} g h_{\sigma}^{-1}=x$ and hence $\kappa(\sigma)(g)=h_{\sigma}{ }^{\sigma} g h_{\sigma}^{-1} \in \bar{G}$. We regard $f_{\sigma}$ as a $\sigma$-semialgebraic automorphism of $\bar{G}$. Then $f: \Gamma_{F} \rightarrow$ SAut $\bar{G}$ is a a continuous map, the composition

$$
\kappa: \Gamma_{F} \rightarrow \operatorname{SAut} \bar{G} \rightarrow \operatorname{SOut} \bar{G}
$$

is a homomorphism and this defines an $F$-kernel $L=(\bar{G}, \kappa)$.

A principal homogeneous space of $H$ over $X$ is a pair $(P, \alpha)$, where $P$ is a right principal homogeneous space of $H$ and $\alpha: P \rightarrow X$ is an $H$-equivariant $F$-morphism. The etale descent shows that $\alpha$ is necessarily faithfully flat.

If $X$ has an $F$-rational point $x_{0}$, then there exists a principal homogeneous space $(P, \alpha)$ over $X$. Indeed, one takes $P=H$ and $\alpha(h)=x_{0} \cdot h$. Conversely, if there exists a principal homogeneous space $(P, \alpha)$ over $X$ and $P$ has an $F$-rational point $p_{0}$, then $X$ has an $F$-rational point $\alpha\left(p_{0}\right)$.

In $[14,1.20]$ (also see $[1,7.7]$ ), Springer defined a cohomology class $\eta(X) \in$ $H^{2}(F, L)$ associated to $X$. Springer [14, 3.7] and Borovoi [1, 7.7, p. 235] proved the following result.

Proposition 5.2. Notations as above. The class $\eta(X)$ is neutral if and only if there exists a principal homogeneous space $(P, \alpha)$ over $X$.

In particular, if $H^{1}(F, H)$ is trivial, then $\eta(X)$ is neutral if and only if $X(F)$ is non-empty.

5.3. Homogeneous spaces of embeddings of $K$ into $A$. We return to the problem of embeddings of an etale algebra $K$ into a central simple algebra $A$. We remain $F$ a global field as in the previous sections. Let $X$ be the $F$-scheme that represents the functor

$$
X(R)=\operatorname{Hom}_{R-a l g}^{*}\left(K \otimes_{F} R, A \otimes_{F} R\right)
$$

for any commutative $F$-algebra $R$. The group $H=A^{\times}$of multiplicative group of $A$, viewed as an algebraic group over $F$, acts naturally on $X$ on the left. We make the right $H$-action on $X$ by setting $x \cdot h:=h^{-1} \cdot x$.

The geometric orbits of $X$ under $H$ are in one-to-one correspondence with the elements in the orbit set $\mathcal{O}_{K \otimes F^{\text {sep }}, A \otimes F^{\text {sep }}}$. Therefore each geometric orbit can be represented by a function $f: \Sigma \rightarrow \mathbb{N}$ on $\Sigma$, where $\Sigma:=\operatorname{Hom}_{F}\left(K, F^{\text {sep }}\right)$. Moreover, this correspondence is $\Gamma_{F}$-equivariant. We have the decomposition of geometric orbits

$$
\bar{X}:=X \otimes_{F} F^{\text {sep }}=\bigcup_{f} \bar{X}_{f},
$$

where each $\bar{X}_{f}$ is a homogeneous space of $\bar{H}:=H \otimes_{F} F^{\text {sep }}$ corresponding to the function $f$. As the correspondence $f \mapsto \bar{X}_{f}$ is $\Gamma_{F}$-equivariant, the subvariety $\bar{X}_{f}$ is defined over $F$ if and only if the function $f$ is $\Gamma_{F}$-invariant.

Let $\bar{X}_{f}$ be a geometric orbit which is defined over $F$. Let $X_{f}$ be the $F$-subscheme of $X$ whose base extension to $F^{\text {sep }}$ is $\bar{X}_{f}$; this is a homogeneous space of $H$ over 
F. As $f$ is $\Gamma_{F}$-invariant, there is a tuple $\left(m_{1}, \ldots, m_{r}\right) \in \mathbb{N}^{r}$ so that for any point $\bar{\epsilon} \in X_{f}\left(F^{\mathrm{sep}}\right)$

$$
\bar{\epsilon}: K \otimes_{F} F^{\text {sep }} \hookrightarrow \bar{A}:=A \otimes F^{\text {sep }}=\operatorname{End}_{F^{\text {sep }}}(\bar{V}),
$$

the induced $K \otimes F^{\mathrm{sep}}$-module $\bar{V}$ is isomorphic to

$$
\left(K_{1} \otimes F^{\mathrm{sep}}\right)^{m_{1}} \oplus \cdots \oplus\left(K_{r} \otimes F^{\mathrm{sep}}\right)^{m_{r}} .
$$

Fix an element $\bar{\epsilon}_{0}$ in $X_{f}\left(F^{\mathrm{sep}}\right)$. The centralizer $\bar{B}$ of the subalgebra $\bar{\epsilon}_{0}\left(K \otimes F^{\mathrm{sep}}\right)$ in $\bar{A}$ is isomorphic to

$$
\operatorname{Mat}_{m_{1}}\left(K_{1} \otimes F^{\mathrm{sep}}\right) \times \cdots \times \operatorname{Mat}_{m_{r}}\left(K_{r} \otimes F^{\mathrm{sep}}\right),
$$

and the stabilizer subgroup $\bar{G}$ of $\bar{\epsilon}_{0}$ is equal to the multiplicative group $\bar{B}^{\times}$(viewed as an algebraic group over $\left.F^{\text {sep }}\right)$. Let $\bar{Z}$ be the center of $\bar{G}$. The center $Z(\bar{B})$ of $\bar{B}$ is equal to $\bar{\epsilon}_{0}\left(K \otimes F^{\text {sep }}\right)$ and we have $\bar{Z}=Z(\bar{B})^{\times}$. The homomorphism $\kappa: \Gamma_{F} \rightarrow$ SOut $\bar{Z}=$ SAut $\bar{Z}$ associated to $X_{f}$ gives an action on the center

$$
\bar{Z}\left(F^{\mathrm{sep}}\right)=Z(\bar{B})^{\times}=\bar{\epsilon}_{0}\left(K \otimes F^{\mathrm{sep}}\right)^{\times} .
$$

LEMMA 5.3.

1. The center $Z$ of $(\bar{G}, \kappa)$ is isomorphic to $\prod_{i=1}^{r} \operatorname{Res}_{K_{i} / F} \mathbb{G}_{\mathrm{m}}$.

2. Any $F$-form $G$ of $\bar{G}$ with $\kappa_{G}=\kappa$ is of the form $\mathrm{GL}_{1}\left(A_{1}\right) \times \cdots \times \mathrm{GL}_{1}\left(A_{r}\right)$ for some central simple $K_{i}$-algebras $A_{i}$ of degree $m_{i}$.

Proof. (1) For any $\sigma \in \Gamma_{F}$, we have ${ }^{\sigma} \bar{\epsilon}_{0}=\operatorname{int}\left(h_{\sigma}^{-1}\right) \circ \bar{\epsilon}_{0}$ for some element $h_{\sigma} \in \bar{A}^{\times}$. We have the commutative diagram:

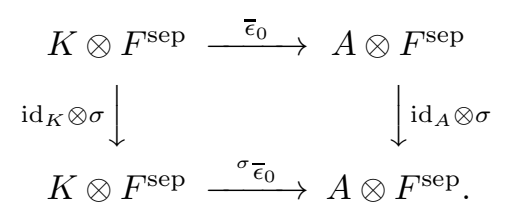

As $\kappa(\alpha)=\operatorname{int}\left(h_{\sigma}\right) \circ\left(\operatorname{id}_{A} \otimes \sigma\right)$, we have the following commutative diagram:

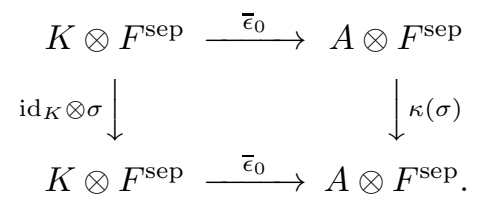

This shows that $\kappa(\sigma)$ acts on $\bar{\epsilon}_{0}\left(K \otimes F^{\mathrm{sep}}\right)$ on the second factor and hence $Z(F)=$ $\bar{\epsilon}_{0}(K)^{\times}$. The $F$-subalgebra generated by $Z(F)$ is equal to $\bar{\epsilon}_{0}(K)$. Therefore, $Z$ is the subtorus $\bar{\epsilon}_{0}(K)^{\times}$and is isomorphic to $\prod_{i=1}^{r} \operatorname{Res}_{K_{i} / F} \mathbb{G}_{\mathrm{m}}$. .

(2) Since the $Z(F)$ generates the $F$-subalgebra $\bar{\epsilon}_{0}(K)$, the $F$-subalgebra generated by $G(F)$ in $\bar{A}$ is a semi-simple $F$-algebra containing $\bar{\epsilon}_{0}(K)$, which is of the form $A_{1} \times \cdots \times A_{r}$, where each $A_{i}$ is a central simple algebra over $\bar{\epsilon}_{0}\left(K_{i}\right)$. Therefore, $G$ is isomorphic to $\mathrm{GL}_{1}\left(A_{1}\right) \times \cdots \times \mathrm{GL}_{1}\left(A_{r}\right)$. This completes the proof of the lemma.

Theorem 5.4 (The Hasse principle). Let $X_{f}$ be a homogeneous space of $H$ defined over $F$ and $\left(m_{1}, \ldots, m_{r}\right)$ be the corresponding tuple as above. If $X_{f}\left(F_{v}\right) \neq \emptyset$ for all $v \in V^{F}$, then $X_{f}(F) \neq \emptyset$. 
Proof. Since $H$ is an inner form of $\mathrm{GL}_{m}$, one has $H^{1}(F, H)=1$ and $H^{1}\left(F_{v}, H\right)=$ 1 by the Hilbert 90 theorem. By Proposition 5.2, the obstruction class $\eta\left(X_{f}\right)$ (resp. $\eta_{v}\left(X_{f}\right)$ ) is neutral if and only if $X_{f}(F) \neq \emptyset\left(\right.$ resp. $\left.X_{f}\left(F_{v}\right) \neq \emptyset\right)$. Choose the quasi-split form $G_{0}=\prod_{i=1}^{r} \operatorname{Res}_{K_{i} / F} \mathrm{GL}_{m_{i}}$ of the isotropic subgroup $\bar{G}$ with $\kappa_{G_{0}}=\kappa$, and let $n\left(G_{0}\right)$ denote the neutral class $\mathrm{Cl}\left(f_{G_{0}}, 1\right) \in H^{2}(F, L)$. As $H^{2}(F, L)$ is non-empty, it is a principal homogeneous space under $H^{2}(F, Z)$; see $[14,1.17$, p. 170]. The base class $n\left(G_{0}\right)$ gives a natural bijection $H^{2}(F, Z) \simeq H^{2}(F, L)$. As $H^{2}(F, Z)=\operatorname{Br}\left(K_{1}\right) \times \cdots \times \operatorname{Br}\left(K_{r}\right)$, the class $\eta\left(X_{f}\right) \in H^{2}(F, L)$ can be represented by $\left(\left[A_{1}\right], \ldots,\left[A_{r}\right]\right) \in H^{2}(F, Z)$, where each $A_{i}$ is a central simple algebra over $K_{i}$. Moreover, the class $\eta(X)$ is neutral if and only if the class $\left(\left[A_{1}\right], \ldots,\left[A_{r}\right]\right)$ lies in the image of the boundary map $H^{1}\left(F, G_{0} / Z\right) \rightarrow H^{1}(F, Z)$ (see [1, Proposition 2.3, p. 224]), that is, the condition

$$
\operatorname{ind}_{K_{i}}\left(A_{i}\right) \mid m_{i}, \quad i=1, \ldots, r
$$

holds. Now under the assumption $X_{f}\left(F_{v}\right) \neq \emptyset$ for all $v \in V^{F}$ we get the condition

$$
\operatorname{ind}_{K_{i, w}}\left(A_{i} \otimes_{K_{i}} K_{i, w}\right) \mid m_{i}
$$

for all $i=1, \ldots, r$ and $w \in V^{K_{i}}$. It follows from the Hasse-Brauer-Noether theorem that $\operatorname{ind}_{K_{i}}\left(A_{i}\right) \mid m_{i}$, for $i=1, \ldots, r$ and hence $X_{f}(F) \neq \emptyset$. This completes the proof of the theorem.

Acknowledgments. The manuscript was revised during the third named author's stay at the IEM, Universität Duisburg-Essen. He wishes to thank the IEM for kind hospitality and excellent working conditions. Yang and Yu were partially supported by grants NSC 97-2115-M-001-015-MY3, 100-2628-M-001-006-MY4 and AS99-CDA-M01. The authors are grateful to the referees for careful readings and helpful comments, specially the suggestion of a referee by bringing into the Hasse principle for homogeneous spaces and his/her kind instruction of the proof of Theorem 5.4.

\section{REFERENCES}

[1] M. Borovor, Abelianization of the second nonabelian Galois cohomology, Duke Math. J., 72 (1993), pp. 217-239.

[2] M. Borovol, The Brauer-Manin obstructions for homogeneous spaces with connected or abelian stabilizer, J. Reine Angew. Math., 473 (1996), pp. 181-194.

[3] M. Borovoi, A cohomological obstruction to the Hasse principle for homogeneous spaces, Math. Ann., 314 (1999), pp. 491-504.

[4] Algebraic Number Theory, edited by W.-S. Cassels and A. Fröhlich. (Proc. Instructional Conf., Brighton, 1965). Academic Press, 366 pp.

[5] P. Chuard-Koulmann And J. Morales, Extending involutions on Frobenius algebras, Manuscripta Math., 108 (2002), pp. 439-451.

[6] M. Eichler, Zur Zahlentheorie der Quaternionen-Algebren, J. Reine Angew. Math., 195 (1955), pp. 127-151.

[7] G. HARDER, Bericht über neuere Resultate der Galoiskohomologie halbeinfacher Gruppen, Jber. Deutsch. Math.-Verein, 70 (1968), pp. 182-216.

[8] R. S. Pierce, Associative algebras, Graduate Texts in Mathematics, 88, Springer-Verlag, New York-Berlin, 1982. 436 pp.

[9] G. Prasad and A. Rapinchuk, Computation of the metaplectic kernel, Inst. Hautes Études Sci. Publ. Math., 84 (1996), pp. 91-187.

[10] G. PRASAD AND A. RAPINCHUK, Local-global principles for embedding of fields with involution into simple algebras with involution, Comment. Math. Helv., 85 (2010), pp. 583-645.

[11] A. S. Rapinchuk, The Hasse principle for symmetric spaces, Dokl. Akad. Nauk BSSR, 31 (1987), pp. 773-776. 
[12] I. Reiner, Maximal orders, London Mathematical Society Monographs, No. 5, Academic Press, London-New York, 1975. 395 pp.

[13] J.-P. SerRe, Local fields, GTM 67, Springer-Verlag, 1979.

[14] T. A. Springer, Nonabelian $H^{2}$ in Galois cohomology, Algebraic Groups and Discontinuous Subgroups, (Proc. Sympos. Pure Math., Boulder, Colo., 1965) pp. 164-182.

[15] M.-F. Vignéras, Arithmétique des algèbres de quaternions, Lecture Notes in Math., vol. 8, Springer-Verlag00, 1980.

[16] C.-F. Yu, Construction of Galois covers of curves with groups of $\mathrm{SL}_{2}$-type, C. R. Acad. Sci. Paris Sér. I Math., 345 (2007), pp. 77-80.

[17] C.-F. YU, Embeddings of fields into simple algebras: generalizations and applications, J. Algebra, 368 (2012), pp. 1-20.

[18] C.-F. YU, Characteristic polynomials of central simple algebras, Taiwanese J. Math., 17 (2013), pp. 351-359. 